\title{
AÇÃO EXTENSIONISTA DE PROMOÇÃO DA SAÚDE PARA ESTUDANTES UNIVERSITÁRIOS
}

\author{
Yago Soares Fonseca \\ Universidade Federal do Sul da Bahia \\ yagosfos@gmail.com \\ Luciane Aparecida Gonçalves Manganelli \\ Universidade Federal do Sul da Bahia \\ lucianemanganelli@gmail.com \\ Grasiely Faccin Borges \\ Universidade Federal do Sul da Bahia \\ grasiely.borges@gmail.com
}

Calila Oliveira Alves Universidade Federal do Sul da Bahia calila_oliv@hotmail.com

Gabriela de Azevedo Barbosa Universidade Federal do Sul da Bahia gabrielabarbosa317@outlook.com

Aline Prates Correia Universidade Federal do Sul da Bahia aline_prates2007@hotmail.com

Adryane Gomes Mascarenhas Universidade Federal do Sul da Bahia adryanemascarenhas@gmail.com

\section{Resumo}

Objetivo: Relatar a experiência e apresentar os resultados de uma ação de promoção da saúde realizada por estudantes na Universidade Federal do Sul da Bahia. Método: A ação configurou-se como semana de atenção à saúde e foram coletados dados antropométricos e cardiovasculares de acordo com as diretrizes do manual técnico da Vigilância Alimentar e Nutricional a VII Diretriz Brasileira de Hipertensão Arterial e atualização da diretriz de prevenção cardiovascular da Sociedade Brasileira de Cardiologia. Resultados: A ação demonstrou que mais de $60 \%$ dos estudantes apresentaram valores adequados para índice de massa corporal, circunferência da cintura e pressão arterial e mais de 40\% para relação cintura-quadril, contudo, uma parcela de 10 a $30 \%$ apresentou algum parâmetro acima do normal. Conclusões: A ação assumiu o caráter de promoção da saúde e tornou o estudante protagonista. Verificou-se que havia estudantes participantes, com necessidade de atenção à saúde.

Palavras-chave: Promoção da Saúde. Serviços de Saúde para Estudantes. Saúde do Estudante. Universidades. Sistema Cardiovascular.

\section{EXTENSIONIST ACTIVITY FOR HEALTH PROMOTION FOR UNIVERSITY STUDENTS}

Abstract

Objective: To report the experience and present the results of a health promotion activity by students at the Federal University of Southern Bahia. Method: The activity was configured as a week of health care and anthropometric and cardiovascular data were collected in accordance with the guidelines of the technical manual of Food and Nutritional Surveillance to the VII Brazilian Guideline for Hypertension and updated Cardiovascular Prevention Guideline of the Brazilian Society of Cardiology. Results: The activity presented that more than $60 \%$ of students had adequate values for body mass index, waist circumference and blood pressure and more than $40 \%$ for waist-to-hip ratio, however, a portion of 10 to 30\% presented some parameters over than normal. Conclusions: The activity assumed the character of health promotion and made the student a protagonist. It was found that there were participating students, in need of health care.

Keywords: Health Promotion. Student Health Services. Student Health. Universities. Cardiovascular System.

\section{ACCIÓN EXTENSIONISTA DE PROMOCIÓN DE LA SALUD PARA ESTUDIANTES UNIVERSITARIOS}

\section{Resumen}

Objetivo: Informar la experiencia y presentar los resultados de una acción de promoción de la salud realizada por um estudiante de la Universidad Federal del Sur de Bahía. Método: La acción se configuró como una semana de atención médica y se recopilaron datos antropométricos y cardiovasculares de acuerdo com lãs directrices del manual técnico de Vigilancia Alimentaria y Nutricional de la VII Guía Brasileña para la Hipertensión y la actualización de laguía de prevención cardiovascular de la Sociedad Brasileña de cardiología. Resultados: la acción mostró que más del $60 \%$ de los Estudiantes tenían valores adecuados para el índice de masa corporal, la circunferencia de la cintura y la presión arterial y más del 40\% para la relación cintura-cadera, sin embargo, una porción del 10 al 30\% presentó algún parámetro por encima de lo normal. Conclusiones: la acción asumió el carácter de promoción de la salud e hizo del alumno un protagonista. Se descubrió que había estudiantes participantes que necesitaban atención a salud.

Palavras clave: Promoción de la Salud. Servicios de Salud para Estudiantes. Salud del Estudiante. Universidades.

Sistema Cardiovascular. 


\section{INTRODUÇÃO}

As Universidades Promotoras da Saúde (UPS) são espaços privilegiados para a criação de um contexto promotor de saúde para a comunidade educativa (Carta de OKANAGAN, 2015). Nessa perspectiva, as ações de extensão universitária atuam, dentre suas diversas esferas, também na promoção da saúde dos estudantes. As UPS assumem particular importância na medida em que se integram à rede assistencial e podem servir de espaço diferenciado para novas experiências voltadas à humanização, ao cuidado e à qualificação da atenção à saúde (HENNINGTON, 2005).

O contexto das UPS faz parte de um conjunto de dinâmicas sociais em que diferentes atores aprendem, trabalham, socializam e desfrutam de seu trabalho ou lazer e serviços disponíveis em favor da saúde individual e coletiva, tornando-se ambientes favoráveis à saúde e ao desenvolvimento sustentável (DOORIS; WILLS; NEWTON, 2014).

O processo saúde-doença é considerado resultante de determinada vivência social e influências diversas, como fatores ligados à instrução tais quais cultura, intelectualidade, escolaridade e, ainda, ligados ao ambiente como saneamento, acesso ao transporte, moradia, água potável e, por fim, o aspecto econômico. Tais fatores influenciam e caracterizam a vida social e a qualidade de vida (LIMA DA SILVA, 2006).

Nessa perspectiva, projetos de extensão podem estimular o autocuidado de forma a sensibilizar o estudante e, além disso, dar autonomia para que tome atitudes no sentido de melhorar a sua qualidade de vida (HENNINGTON, 2005; LIMA DA SILVA, 2006). Nas universidades onde há intervenções de saúde, como ações educativas e projetos de extensão, constatam-se melhoras no perfil de saúde do estudante através do desenvolvimento da educação, da pesquisa e do compartilhamento de conhecimentos, o que contribui para o bemestar e sustentabilidade da comunidade como um todo (FERREIRA; DA SILVA BRITO; SANTOS, 2018).

A importância da realização de ações de promoção da saúde para estudantes universitários também é justificada pelo fato de, no Brasil, as Doenças Crônicas não Transmissíveis constituírem a principal causa de morte em adultos, com destaque para as doenças cardiovasculares (DCV), diabetes, neoplasias e doenças respiratórias crônicas (BRASIL, 2008).

De acordo a atualização da Diretriz de Prevenção Cardiovascular da Sociedade Brasileira de Cardiologia (PRÉCOMA et al., 2019), o Brasil ocupa, atualmente, o quarto lugar entre os países com maior prevalência de obesidade. Entre o período de 1980 a 2013, a 
Ação extensionista de promoção da saúde de estudantes universitários

quantidade de pessoas que ultrapassaram o IMC maior que $25 \mathrm{Kg} / \mathrm{m}^{2}$ subiu de 28,8 para 36,9\%.Além disso, em 2018, a incidência de homens com sobrepeso, a partir de 20 anos, atingiu 55,8\% enquanto que os índices de obesidade foram o equivalente a 18,7\%, sendo que, em mulheres, estes índices chegaram a 53,9\% e 20,7\%, respectivamente. O ganho de peso está estritamente associado a mudanças nos padrões alimentares e à prática de atividade física, sendo que estas mudanças são decorrentes das transformações demográficas, econômicas e sociais vigentes (PRÉCOMA et al., 2019).

A realização de monitoramento, informação e educação sobre os fatores de risco constituem, para além de prevenção de doenças, a promoção da saúde do universitário. Esse tipo de ação representa uma alternativa essencial para mudanças de hábitos alimentares, perfil antropométrico e estilo de vida nesse período que marca a transição da adolescência para a vida adulta e o ingresso no mercado de trabalho, por meio de abordagens com apelo motivacional, como descrito pelo estudo de Berbigier e Magalhães (2017), que analisou artigos produzidos em diferentes países acerca das estratégias de promoção da saúde para estudantes universitários. Dessa maneira, a dedicação de tempo extracurricular na universidade é reconhecida como uma fase importante para promover a saúde dos alunos e prevenir doenças (NELSON et al., 2008).

A não adoção de hábitos saudáveis em consequência do ingresso na universidade pode ser explicada pelo surgimento de novas possibilidades de adoção de comportamentos sedentários e alimentação inadequada, favorecendo o sobrepeso e/ou obesidade. Em decorrência disso há uma prevalência do excesso de peso, aumento da circunferência da cintura e fortes preditores de hipertensão arterial nesse público, sendo a obesidade central um importante fator de risco cardiovascular (PIRES; MUSSI, 2016).Essa realidade ainda interfere diretamente na percepção do indivíduo sobre si de forma positiva ou negativa. Os indivíduos que avaliam sua própria saúde julgam a sua situação física, mental e social em geral e, frequentemente, concordam com a avaliação feita por médicos, representando um importante indicador, pois o nível de bem-estar dos indivíduos pode influenciar a sua qualidade de vida (SZWARCWALD et al., 2015).

As práticas de promoção da saúde no ambiente acadêmico estão sendo aplicadas em todo o mundo, elas têm mostrado que os espaços promotores da saúde são contextos de intervenção em que estratégias multiníveis são dinamizadas e o conhecimento é mobilizado para a promoção de ambientes e comportamentos saudáveis (BERBIGIER; MAGALHÃES, 2017). 
Ação extensionista de promoção da saúde de estudantes universitários

Embora os estudos no Brasil acerca do potencial ambiente de promoção da saúde que é a universidade ainda sejam incipientes, o país vem, por meio das UPS, subsidiar ambientes promotores de saúde dentro da comunidade acadêmica, os quais, a partir da educação em saúde, métodos dialógicos e formas criativas de realizar a promoção e mobilização comunitária, vêm facilitando o processo de autonomia do estudante para o controle de sua saúde e bemestar, contribuindo para a literacia em saúde, adoção de hábitos e condições favoráveis à saúde e melhoria do ambiente físico e social (FERREIRA; DA SILVA BRITO; SANTOS, 2018).

Mudanças de comportamento na fase universitária são caracterizadas pela mudança no estilo de vida (SOUZA; BORGES, 2016), entre elas estão a adoção de comportamentos de risco, como consumo de bebidas alcóolicas (FRANCA; COLARES, 2008; PEDROSA et. al., 2011; FEITOSA et al., 2010; SAWICKI et al., 2018), baixos níveis de atividade física (DE SOUZA; NAHAS, 2011; FONTES; VIANNA, 2009) e alimentação inadequada (DUARTE, 2013). Quando os jovens ingressam na universidade, muitas vezes se afastam do seu círculo de relacionamentos familiares e sociais, o que pode desencadear situações de crise e busca de estratégias para enfrentar essa situação (PELICIOLI et al., 2017).

Muitos estudantes nas universidades públicas brasileiras são oriundos de classes populares, apresentando demandas de compreensão da saúde na interação do ensino superior e os aspectos sociais, econômicos e pessoais (OLIVEIRA; PADOVANI, 2014). Na região Nordeste do Brasil,são preocupantes os padrões relacionados à saúde apresentados por estudantes universitários com relação aos hábitos alimentares, prática de atividades físicas, tabagismo e consumo de bebidas alcoólicas (SOUSA; JOSÉ; BARBOSA, 2013). Estudo demonstra que a vida universitária e o seu contexto alimentar estão frequentemente associados a hábitos alimentares inadequados nos estudantes (BERNARDO, et al., 2017).

Trabalhos anteriormente realizados visando à promoção de saúde em adolescentes em idade escolar indicam que nas regiões Sul, Sudeste, Nordeste e Centro-oeste a prevalência da hipertensão arterial é de 8\% (GONÇALVES et al., 2016). Além da hipertensão arterial, a obesidade também tem índice crescente nos indivíduos do ensino superior, embora apresente maior prevalência entre pessoas com menor nível de escolaridade e outra comorbidade que afeta cada vez mais adolescentes e adultos jovens é a diabetes tipo II (BAILEY et al., 2013).

Assim, a inserção no âmbito acadêmico é marcada por mudanças físicas, comportamentais e sociais, envoltas em novas vivências e hábitos, que comprometem, em sua maioria, a saúde do universitário. As UPS estabelecem, dessa forma, métodos intersetoriais motivacionais que contribuem para a promoção da saúde, adoção de hábitos saudáveis e melhora da qualidade de vida dentro desses espaços. 
Ação extensionista de promoção da saúde de estudantes universitários

Nesse contexto, a realização do projeto se constituiu como uma importante ferramenta de intervenção de informação e educação em saúde, ampliação do autocuidado e autopercepção, bem como melhoria da saúde dos estudantes envolvidos, tanto para os que executaram a proposta quanto para quem participou da mesma. Ainda, o relato dessa ação de promoção da saúde permite observar o perfil antropométrico e parâmetros cardiovasculares de parte dos estudantes em uma instituição pública e, para além de tudo, inspirar outras ações do mesmo cunho que revelem o potencial que o ambiente universitário tem em se transformar em promotor da saúde, uma vez que os estudantes passam uma parcela considerável de sua vida neste lugar.

Dessa forma, esse trabalho objetiva relatar a experiência e apresentar os resultados de uma ação de promoção da saúde realizada na Universidade Federal do Sul da Bahia - Campus Paulo Freire denominada "Semana de atenção à saúde do estudante universitário e prevenção de comorbidades".

\section{MATERIAL E MÉTODOS}

Trata-se de um relato de experiência de uma ação de extensão universitária, denominada "Semana de atenção à saúde do estudante universitário e prevenção de comorbidades", desenvolvida por acadêmicos da UFSB dos cursos de Bacharelado Interdisciplinar em Saúde e Medicina.

A atividade extensionista surgiu a partir do projeto da UFSB denominado Universidade Promotora da Saúde, financiada pelo Governo Federal por meio do decreto $n^{\circ} 7.234 / 2010$, com o objetivo de selecionar propostas de intervenção na área da Promoção da Saúde. Ocorreu na Universidade Federal do Sul da Bahia-Campus Paulo Freire, em Teixeira de Freitas, Bahia, no período de 12 a 14 de novembro de 2018.

O público alvo da ação foram os estudantes universitários. Para participar, o estudante deveria estar matriculado em algum curso oferecido pela UFSB-Campus Paulo Freire, de primeiro ciclo, como os Bacharelados Interdisciplinares, ou de segundo ciclo, como os cursos de Medicina e Psicologia. Foram excluídos os participantes com idade menor que 18 anos, bem como os que se recusaram a participar de alguma das estações de obtenção das medidas ou que não concordaram com algum procedimento adotado.

A divulgação da ação foi realizada por meio de folders digitais enviados para o e-mail de todos os estudantes com apoio da Secretaria Acadêmica da UFSB-Campus Paulo Freire. 
Ação extensionista de promoção da saúde de estudantes universitários

Para realizar a ação foram montadas duas estruturas em uma área externa de convivência do campus, com uma mesa e duas cadeiras cada, e uma sala foi reservada, formando assim três estações. A quarta estação não utilizou mesa nem cadeira. Em cada estação, uma cadeira foi destinada para acomodar o estudante executor e outra para o estudante participante, a mesa foi utilizada para colocar objetos utilizados na ação como os medidores de pressão, notebooks, fitas métricas, dentre outros. Cada participante recebeu um pequeno papel onde foram anotados os valores obtidos à verificação de Pressão Arterial (PA) e Frequência Cardíaca (FC), medidas antropométricas, bem como o resultado das relações feitas entre elas. Cada estudante executor realizou apenas uma ação na estação, sendo que estas foram divididas do seguinte modo:

$1^{a}$ Estação: nesta estação, o estudante executor aferiu a pressão arterial e a frequência cardíaca dos participantes com um medidor de pressão arterial automático de braço da marca Omrom Hem 7122. Para classificação dos níveis pressóricos foi utilizada a VII Diretriz Brasileira de Hipertensão Arterial (MALACHIAS et al., 2016), que classifica como normotensos aqueles com pressão arterial sistólica (PAS) menor ou igual a $120 \mathrm{mmHg}$ e pressão arterial diastólica (PAD) menor ou igual a $80 \mathrm{mmHg}$; pré-hipertensos aqueles com PAS entre 121 e 139 mmHg e PAD entre 81 e 89 mmHg; hipertensão estágio I, os indivíduos com PAS entre 140 e 159 mmHg e PAD entre 90 e 99 mmHg; hipertensão estágio II, aqueles com níveis de PAS entre 160 e 179 mmHg e PAD entre 100 e 109 mmHg; e, hipertensão estágio III, quando PAS maior ou igual a $180 \mathrm{mmHg}$ e PAD maior ou igual a $110 \mathrm{mmHg}$ (PRÉCOMA et al., 2019). Em relação à frequência e ritmo da onda P sinusal, os participantes foram classificados como normocárdicos se FC foi entre 50 e 100 batimentos por minuto (bpm); taquicardia sinusal se FC maior do que 100 bpm; e, bradicardia sinusal se FC menor do que $50 \mathrm{bpm}$, de acordo com os parâmetros de Pastore et al. (2016).

$2^{a}$ Estação: essa etapa foi realizada em ambiente fechado, na sala reservada, pois foi necessário que o participante levantasse a blusa. Utilizou-se uma fita métrica comum de $150 \mathrm{~cm}$ para obtenção das medidas de Circunferência da Cintura (CC) e Circunferência do Quadril (CQ). Após obtidas tais medidas, foi calculada a Relação Cintura Quadril (RCQ) por meio da divisão do perímetro da cintura $(\mathrm{cm})$ pelo perímetro do quadril $(\mathrm{cm})$. Os valores de referência de CC para mulheres é menor que $80 \mathrm{~cm}$ e, para homens, menor que $94 \mathrm{~cm}$; já os valores de RCQ para mulheres devem ser menores que 0,85 e, para homens, menores que 0,9 (WHO, 2000).

$3^{a}$ Estação: nesta, foram obtidas medidas de peso e altura dos participantes com uma balança antropométrica digital adulto da marca Balmak com capacidade para 200kg. Esses 
dados foram utilizados para calcular o Índice de Massa Corporal (IMC), dividindo-se o peso pelo quadrado de sua altura. Para a classificação, foram utilizados os parâmetros adotados pela Organização Mundial da Saúde, sendo definida do seguinte modo: baixo peso (IMC menor que $18,5 \mathrm{Kg} / \mathrm{m}^{2}$ ); peso ideal (IMC entre 18,5 e 24,9 Kg/m²); sobrepeso (IMC entre 25,0 e 29,9 $\mathrm{Kg} / \mathrm{m}^{2}$ ); ou obesidade (IMC maior ou igual a $\left.30,0 \mathrm{~kg} / \mathrm{m}^{2}\right)(\mathrm{WHO}, 2000)$.

$4^{a}$ Estação: nesta última estação os participantes receberam orientações de cuidado com a saúde para prevenção de doenças e comorbidades e passaram por uma sessão de 3 pôsteres. O primeiro pôster continha silhuetas que representavam cada IMC, no segundo constava a classificação de risco para desenvolvimento de DCV de acordo resultado da RCQ, levando em consideração sexo e idade e, por fim, um terceiro pôster com fluxograma para identificação do risco de desenvolvimento de eventos cardiovasculares futuros de acordo os fatores de risco apresentados.

Os materiais utilizados foram cedidos pelo Núcleo de Estudos e Pesquisas em Saúde NEPS e também pelo Setor de Saúde, ambos do Campus Paulo Freire da UFSB. A coleta das medidas antropométricas seguiu as determinações das diretrizes do manual técnico da Vigilância Alimentar e Nutricional (SISVAN) que fornece orientações básicas para a coleta, o processamento, a análise de dados e a informação em serviços de saúde (BRASIL, 2011). Para uma coleta precisa dos dados antropométricos de um indivíduo adulto, a SISVAN estabelece algumas orientações: a) peso: indivíduos devem ser pesados descalços, com roupas leves, posicionados de costas para a balança, no centro, ereto, pés juntos e braços rentes ao corpo, mantê-lo parado nessa posição; b) altura: indivíduos devem estar descalços, em pé, no centro, ereto, com a cabeça livre de adereços, braços rentes ao corpo e pés paralelos em ângulo reto com as pernas, cabeça fixa, olhando para um ponto fixo na altura dos olhos, no plano de Frankfurt; e c) CC: indivíduo deve estar em pé, ereto, abdômen relaxado, braços rentes ao corpo e pernas paralelas e moderadamente separadas, roupas e cintos devem ser removidos ou afastados.

Todas as medidas e avaliações realizadas foram tabuladas em planilhas eletrônicas no EXCEL e passaram por dupla digitação para conferência de inconsistências e reunidas como uma única amostra. Para apresentação das variáveis, utilizou-se média e desvio padrão em tabelas.

\section{RESULTADOS E ANÁLISES}


Ação extensionista de promoção da saúde de estudantes universitários

A extensão universitária é promotora de interação transformadora mediada por alunos, por meio do estímulo para o desenvolvimento social e o espírito crítico nos estudantes participantes da ação, contribuindo também para a melhoria na qualidade da educação por meio do contato dos extensionistas com as realidades concretas na promoção da saúde e na avaliação do estado de saúde dos participantes (RABELO et al., 2016).

Participaram da Semana de Atenção à Saúde do Estudante Universitário 80 estudantes de ambos os sexos dos diversos cursos da UFSB no Campus Paulo Freire. No momento da ação, 912 estudantes estavam matriculados. Destes, 578 eram alunos do primeiro ciclo, sendo 311 do sexo feminino e 267 do sexo masculino, e no segundo ciclo, havia 334 alunos, sendo 223 do sexo feminino e 111 do sexo masculino.

Dos 80 participantes da ação, 67,5\% $(n=54)$ eram do sexo feminino e 32,5\% ( $n=26)$ do sexo masculino, com idade média de 22,96 anos. Culturalmente, a concepção de cuidado e a busca mais assídua por serviços de saúde são atribuídas às mulheres (MOREIRA; GOMES; DOS SANTOS, 2010). Um estudo, entretanto, demonstrou que a prevalência de fatores de risco cardiovasculares entre homens jovens é grande (EYKENet al., 2009).

A tabela 1 mostra que as médias de peso $(\mathrm{Kg})$ e estatura $(\mathrm{cm})$ foram, respectivamente, de $58,5 \mathrm{Kg}$ e $161 \mathrm{~cm}$ para mulheres e de $72,1 \mathrm{Kg}$ e $173 \mathrm{~cm}$ para homens. Sabe-se que o peso corporal e a estatura têm forte influência genética e são variáveis passíveis de fortes interferências sociais de padrões de beleza (DAMASCENO, 2008), dessa forma, não é possível neste estudo mensurar tais medidas ideais.

Tabela 1 - Variáveis antropométricas e cardiovasculares de estudantes universitários $(n=80)$ atendidos na "Semana de atenção à saúde do estudante universitário e prevenção de comorbidades" -UFSB.

Média

(feminina $\mathrm{n}=54)$

Variável

Peso $(\mathrm{Kg})$

$58,5( \pm 8,6)$

Estatura $(\mathrm{cm})$

$161,0( \pm 0,05)$

$\operatorname{IMC}\left(\mathrm{kg} / \mathrm{cm}^{2}\right)$

$22,44( \pm 3,3)$
$72,1( \pm 12,7)$

$62,2( \pm 11,9)$

$173,0( \pm 0,07)$

$164,0( \pm 0,08)$

Média total

( masculina $\mathrm{n}=26$ ) 

$\mathrm{CC}(\mathrm{cm})$
$69,9( \pm 5,8)$
$81,0( \pm 9,4)$
$73,5( \pm 8,8)$
CQ (cm)
$94,8( \pm 8,4)$
$99,8( \pm 8,3)$
$96( \pm 8,4)$
RCQ
$0,7( \pm 0,04)$
$0,8( \pm 0,04)$
$0,7( \pm 0,06)$
PAS (mmHg)
$111,3( \pm 9,8)$
$127,4( \pm 9,9)$
$117,1( \pm 14,0)$
PAD (mmHg)
$66,5( \pm 11,1)$
$69,5( \pm 8,2)$
$68,1( \pm 10,7)$
FC (bpm)
$80,7( \pm 12,5)$
$79,6( \pm 15,7)$
$80,3( \pm 13,5)$
IMC: índice de massa corporal; RCQ: relação cintura/quadril; PAS: pressão arterial sistólica; PAD: pressão arterial diastólica; CC: Circunferência da Cintura; CQ: Circunferência do Quadril
Fonte: elaborado pelos autores, 2019.

Para o sexo feminino, 9,25\% apresentaram baixo peso, 68,5\% peso ideal e 21,42\% sobrepeso, e para o sexo masculino 7,6\% apresentaram baixo peso, 53,8\% peso ideal, 30,7\% sobrepeso e 7,6\% obesidade. Do total de participantes 8,75\% apresentavam baixo peso, $63,75 \%$ peso ideal, $25 \%$ sobrepeso, e 2,5\% obesidade, esta última diferente do estudo realizado entre estudantes de enfermagem de uma universidade pública de Salvador, em que houve prevalência de 4,5\% para obesidade I e II (PIRES; MUSSI, 2016). Ressalta-se, também, a pesquisa realizada por Sousa e Barbosa (2017), em que foi identificado aumento da prevalência de excesso de peso corporal em universitários de uma instituição baiana entre os anos de 2012 a 2014.

Em relação às medidas antropométricas apresentadas na tabela 1, 87,5\% do total de participantes da ação apresentaram valores de CC dentro do esperado (para mulheres menor que $80 \mathrm{~cm}$ e para homens menor que $94 \mathrm{~cm}$ ), de acordo com sexo e a idade, enquanto 12,5\% dos acadêmicos (9,2\% do sexo feminino e $11,5 \%$ do sexo masculino) foram classificados com algum grau de risco. Os resultados são similares ao de outros estudos recentes realizados em universitários, com a média dos valores de CC dentro do padrão tanto para homens quanto para mulheres (CARVALHO et al., 2015; GASPARETTO et al., 2012; CORREIA; CAVALCANTE; SANTOS, 2010). 
Ação extensionista de promoção da saúde de estudantes universitários

Quanto ao item RCQ foi possível observar que a maioria dos estudantes (43,75\%) mantiveram suas medidas dentro dos padrões normais. No entanto, uma mulher apresentavase no limite do corte de risco para o desenvolvimento de DCV, o que denota a importância do desenvolvimento de ações que visem estimular a manutenção da saúde por meio da promoção de hábitos de vida saudáveis.

Em relação aos níveis médios de PA, 95\% dos participantes foram classificados como normotensos (com médias de PAS menor ou igual a $120 \mathrm{mmHg}$ e PAD menor ou igual a 80 $\mathrm{mmHg}$ ) e $5 \%$ (3,7\% do sexo feminino e 7,6\% do sexo masculino) foram classificados como pré-hipertensos (PAS entre 121 e 139 mmHg e PAD entre 81 e $89 \mathrm{mmHg}$ ). 11,5\% dos homens se enquadraram em hipertensão sistólica isolada estágio I. De acordo aos níveis pressóricos, a VII Diretriz Brasileira de Hipertensão (MALACHIAS et al., 2016) e a atualização da diretriz de prevenção cardiovascular da Sociedade Brasileira de Cardiologia (PRÉCOMA et al. 2019) classifica esses participantes em baixo risco de desenvolvimento de eventos cardiovasculares futuros.

A avaliação da FC, de acordo com os parâmetros de Pastore et al. (2016), evidenciou que $91,25 \%$ dos indivíduos participantes foram considerados normocárdicos; $1,25 \%$ como taquicárdicos e nenhum participante apresentou bradicardia sinusal. A FC média total foi de 79,4 bpm. Os resultados obtidos estão em conformidade com os achados de um estudo realizado com estudantes do curso de fisioterapia do Centro Universitário Lusíada no estado de São Paulo com idade média de 26,95 anos. Dentre 90 estudantes avaliados, em 10 (11,11\%) foram encontrados níveis de hipertensão arterial (estágio I) e a FC média total foi de 78,3 bpm (CORREIA; CAVALCANTE; SANTOS, 2010).

De forma geral, observa-se que a maior parcela dos estudantes que participaram da ação apresentaram parâmetros antropométricos e cardiovasculares dentro da normalidade, de acordo com as referências utilizadas, entretanto, uma parcela de 10 a 30\% dos estudantes apresentaram valores acima dos considerados ideais, o que indica a necessidade de maior atenção à saúde. Dessa forma, ações extensionistas que visem à promoção da saúde, configuram-se como ferramentas de informação, orientação e incentivo a hábitos saudáveis que melhorem a qualidade de vida dos estudantes, evitando agravos no quadro de saúde desses indivíduos (PRÉCOMA., 2019).

Durante a ação, na estação 4, momento das orientações em que foram mostrados os pôsteres de orientação e cuidados em saúde, tornou-se perceptível que para que ferramentas como as ações de promoção da saúde continuem tendo impacto na vida dos indivíduos, faz-se necessária uma boa comunicação em saúde, a aproximação para o contexto do acadêmico 
Ação extensionista de promoção da saúde de estudantes universitários

acerca do significado de restringir hábitos em prol da melhora de sua qualidade de vida, de forma clara, simples e objetiva.

Além disso, a educação e comunicação em saúde contribuem para o desenvolvimento da responsabilidade individual e prevenção de agravos por meio da transformação de práticas e comportamentos individuais, haja vista que nos países em desenvolvimento, como o Brasil, os adultos jovens são especialmente vulneráveis às doenças crônicas, pois estão mais expostos a riscos por dificuldades econômicas, fatores ambientais e sociais, tendendo a desenvolver doenças cada vez mais jovens, frequentemente com complicações (OMS, 2005).

Outras experiências relatadas demonstram êxito nas ações educativas em saúde para os estudantes de uma escola estadual em Minas Gerais, desenvolvidas por acadêmicos de enfermagem da Pontifícia Universidade Católica de Minas Gerais, onde foram atendidos alunos que tiveram realizadas aferições de pressão arterial, medições de glicemia e cálculos de índice de massa corporal (RANGEL et al., 2016). Da mesma forma, o estudo de Correa et al. (2015), valoriza os projetos extensionistas de vivências em saúde desenvolvidos nas escolas por irem ao encontro de seus princípios educacionais, contribuindo para a formação desses sujeitos, no sentido do estímulo à promoção da saúde e da chamada ao comprometimento com seu bem-estar.

Portanto, as ações extensionistas que têm como foco a promoção da saúde são importantes ferramentas que possibilitam a superação do pensamento estritamente biomédico e curativo, e torna os indivíduos e o espaço em que estão inseridos, potenciais promotores de saúde e multiplicadores dos conhecimentos adquiridos.

\section{CONSIDERAÇÕES FINAIS}

Como resultado da ação, observa-se que a maior parcela dos participantes apresentou valores antropométricos e cardiovasculares dentro do esperado, de acordo os parâmetros da Organização Mundial da Saúde e da Sociedade Brasileira de Cardiologia, entretanto, uma pequena parcela ainda necessitava de atenção à saúde. Dessa forma, a ação assume ainda um papel importante e incentivador da continuidade do autocuidado e do autoconhecimento para manutenção da saúde.

Estratégias de promoção de saúde devem estar presentes em uma rotina universitária com maior frequência, uma vez que os universitários são expostos a diversos fatores de risco. Além disso, é importante que a participação dos estudantes seja motivada pela própria 
Ação extensionista de promoção da saúde de estudantes universitários

universidade, com a disposição e organização de horários específicos para a troca de ideias, uma vez que este é um ambiente propício para abrir caminhos de mudanças na realidade social.

\section{AGRADECIMENTOS}

Agradecemos aos demais integrantes do Núcleo de Estudos e Pesquisas em Saúde da Universidade Federal do Sul da Bahia - Ana Carolina Oliveira Carvalho, Carol Gonçalves Pinto, Gabriel Almeida Santos, Gabriel Santos Costa da Cruz, Marina Lima de Oliveira Carvalho, Murilo Sousa Ramos, Guilherme Paranhos Goulart e Luiz Carlos Cardoso da Silva Junior - que auxiliaram na execução da ação e na coleta de dados. Agradecemos ainda à Universidade Federal do Sul da Bahia por oportunizar os estudantes em vivências com novas perspectivas de promoção da saúde e promovê-la junto a seus pares e que outras universidades possam se espelhar nessa prática, consolidando assim um campo de práticas de promoção da saúde dentro do ambiente universitário.

\section{REFERÊNCIAS}

BAILEY, Clifford J. et al. Individualized glycaemic targets and pharmacotherapy in type 2 diabetes. Diabetes and Vascular DiseaseResearch, v. 10, n. 5, p. 397-409, 2013.

BERBIGIER, Marina Carvalho; MAGALHÃES, Cleidilene Ramos. Educação nutricional em universitários e estratégias para promoção de saúde institucional: revisão integrativa. Revista Brasileira em Promoção da Saúde, v. 30, n. 2, 2017.

BERNARDO, Greyce Luci et al. Food in take of university students. Revista de Nutrição, v. 30, n. 6, p. 847-865, 2017.

BRASIL. Ministério da Saúde. Diretrizes e recomendações para o cuidado integral de doenças crônicas não transmissíveis: promoção da saúde, vigilância, prevenção e assistência. Brasília: Ministério da Saúde; 2008.

BRASIL, Ministério da Saúde. Secretaria de atenção à saúde. Departamento de atenção básica. Orientações para a coleta e análise de dados antropométricos em serviços de saúde: Norma Técnica do Sistema de Vigilância Alimentar e Nutricional-SISVAN. 2011.

CARTA DE OKANAGAN. AnInternational Charter For Health PromotingUniversities\&Colleges. Canadá, 2015. Disponível em: https://open.library.ubc.ca/cIRcle/collections/53926/items/1.0132754. Acesso em 13 de mar de 2019. 
CARVALHO, Carolina Abreu de et al. Associação entre fatores de risco cardiovascular e indicadores antropométricos de obesidade em universitários de São Luís, Maranhão, Brasil. Ciência \& Saúde Coletiva, v. 20, p. 479-490, 2015.

CORRÊA, Priscila Milano et al. Vivências em promoção da saúde: Articulando saberes com estudantes de escolas públicas.Extensio: Revista Eletrônica de Extensão, v. 12, n. 19, p. 14-24, 2015.

CORREIA, Beatriz Rolim; CAVALCANTE, Elder; SANTOS, E. dos. A prevalência de fatores de risco para doenças cardiovasculares em estudantes universitários.RevBrasClinMed, v. 8, n. 1, p. 25-29, 2010.

DAMASCENO, Vinícius Oliveira et al. Imagem corporal e corpo ideal. Revista brasileira de ciência e movimento, v. 14, n. 2, p. 81-94, 2008.

DE SOUZA, Thiago Ferreira; NAHAS, Markus Vinicius. Prevalência e fatores associados a menores níveis de prática de atividades físicas no lazer em estudantes de uma universidade pública do Estado da Bahia. Revista Brasileira de Atividade Física \& Saúde, v. 16, n. 4, p. 322-329, 2011.

DOORIS, Mark; WILLS, Jane; NEWTON, Joanne. Theorizing healthy settings: a critical discussion with reference to Healthy Universities. Scandinavianjournalofpublichealth, v. 42, n. 15_suppl, p. 7-16, 2014.

DUARTE, Flávia Moreno; ALMEIDA, Suzy Darlen Soares de; MARTINS, Karine Anusca. Alimentação fora do domicílio de universitários de alguns cursos da área da saúde de uma instituição privada. O mundo da Saúde, v. 37, n. 3, p. 288-298, 2013.

EYKEN, Elisa Beatriz Braga Dell'Orto et al. Prevalência de fatores de risco para doenças cardiovasculares entre homens de uma população urbana do Sudeste do Brasil. Cadernos de Saúde Pública, v. 25, p. 111-123, 2009.

FEITOSA, Eline Prado Santos et al. Hábitos alimentares de estudantes de uma universidade pública no Nordeste, Brasil Food habits of students of one public university of Northeast, Brazil. Alimentos e Nutrição Araraquara, v. 21, n. 2, p. 225-230, 2010.

FERREIRA, Fernanda Maria Príncipe Bastos; DA SILVA BRITO, Irma; SANTOS, Margarida Reis. Programas de promoção da saúde no ensino superior: revisão integrativa de literatura. Revista Brasileira de Enfermagem, v. 71, 2018.

FONTES, Ana Cláudia Dias; VIANNA, Rodrigo Pinheiro Toledo. Prevalência e fatores associados ao baixo nível de atividade física entre estudantes universitários de uma universidade pública da região Nordeste - Brasil. Rev. bras. epidemiol., São Paulo , v.12, n.1, p.20-29, Mar. 2009.

FRANCA, Carolina da; COLARES, Viviane. Estudo comparativo de condutas de saúde entre universitários no início e no final do curso. Revista de Saúde Pública, v. 42, p. 420427, 2008. 
Ação extensionista de promoção da saúde de estudantes universitários

GASPARETTO, Rosa Maria et al. Perfil antropométrico dos universitários dos cursos de nutrição, enfermagem, fisioterapia e educação física do Centro Universitário La Salle, Canoas/RS. Revista da Associação Brasileira de Nutrição-RASBRAN, v. 4, n. 1, p. 29-33, 2012.

GONÇALVES, Vivian Siqueira Santos et al. Prevalência de hipertensão arterial entre adolescentes: revisão sistemática e metanálise. Revista de Saúde Pública, v. 50, p. 27-27, 2016.

HENNINGTON, Élida Azevedo. Acolhimento como prática interdisciplinar num programa de extensão universitária. Cadernos de Saúde Pública, v. 21, p. 256-265, 2005.

LIMA DA SILVA, J. L. Educação em saúde e promoção da saúde: a caminhada dupla para a qualidade de vida do cliente. Informe-se em promoção da saúde, v. 1, n. 1, p. 03, 2006.

MALACHIAS, Marcus Vinícius Bolívar et al. $7^{\text {a }}$ Diretriz brasileira de hipertensão arterial. ArqBrasCardiol, v. 107, n. 3, p. 1-103, 2016.

MOREIRA, Thereza Maria Magalhães; GOMES, Emiliana Bezerra; DOS SANTOS, Jênifa Cavalcante. Fatores de risco cardiovasculares em adultos jovens com hipertensão arterial e/ou Diabetes Mellitus. Revista Gaúcha de Enfermagem, v. 31, n. 4, p. 662, 2010.

NELSON, Melissa C. et al. Emerging adult hood and college age dyouth: an over looked age for weight related behavior change.Obesity, v. 16, n. 10, p. 2205-2211, 2008.

OLIVEIRA, Nara Rejane Cruz de; PADOVANI, Ricardo Da Costa. Saúde do estudante universitário: uma questão para reflexão. Ciência \& Saúde Coletiva, v. 19, p. 995-996, 2014.

Organização Mundial da Saúde, Organização PanAmericana da Saúde. Prevenção de doenças crônicas: um investimento vital. Brasília (DF); 2005.

PASTORE, C. A. et al. III Diretrizes da Sociedade Brasileira de Cardiologia sobre análise e emissão de laudos eletrocardiográficos. ArqBra. deCardiol, v.106, n.4, supl.1. Abril, 2016.

PEDROSA, Adriano Antonio da Silva et al. Consumo de álcool entre estudantes universitários. Cadernos de Saúde Pública, v. 27, p. 1611-1621, 2011.

PELICIOLI, Marina et al. Perfil do consumo de álcool e prática do beber pesado episódico entre universitários brasileiros da área da saúde. Jornal Brasileiro de psiquiatria, v. 66, n. 3, p. 150-6, 2017.

PIRES, Cláudia Geovana da Silva; MUSSI, Fernanda Carneiro. Excessweight in nursingjuniorandseniorundergraduatestudents. Escola Anna Nery, v. 20, n. 4, 2016.

PRÉCOMA, Dalton Bertolimet al. Atualização da Diretriz de Prevenção Cardiovascular da Sociedade Brasileira de Cardiologia-2019. Arquivos Brasileiros de Cardiologia, v. 113, n. 4, p. 787-891, 2019. 
Ação extensionista de promoção da saúde de estudantes universitários

RABELO, Marcella Oliveira et al. O mutirão de prevenção ao câncer: um relato de experiência no âmbito da extensão universitária. Revista Intercâmbio, v. 7, p. 406-413, 2016.

RANGEL, Selma Maués et al. Ação extensionista de acadêmicos de enfermagem em escola pública: educação e promoção em saúde na comunidade. Enfermagem Revista, v. 16, n. 2, p. 149-158, 2016.

SAWICKI, Wanda Cristina et al. Alcohol consumption, Quality of Life and Brief Intervention among Nursing university students. Revista brasileira de enfermagem, v. 71, p. 505-512, 2018.

SOCIEDADE BRASILEIRA DE CARDIOLOGIA (SBC). $7^{\circ}$ Diretriz Brasileira de Hipertensão Arterial. Arquivos Brasileiros de Cardiologia. São Paulo, v. 107, n. 3, supl. 3, p. 1-83, Set 2016.

SOUSA, Thiago Ferreira de; JOSÉ, Helma Pio Mororó; BARBOSA, Aline Rodrigues. Condutas negativas à saúde em estudantes universitários brasileiros. Ciência \& Saúde Coletiva, v. 18, p. 3563-3575, 2013..

SOUSA, Thiago Ferreira de; BARBOSA, Aline Rodrigues. Prevalências de excesso de peso corporal em universitários: análise de inquéritos repetidos. Revista Brasileira de Epidemiologia, v. 20, p. 586-597, 2017.

SOUSA, Keroléen Jamile Queiroz de; BORGEs, Grasiely Faccin. Estilo de vida, atividade física e coeficiente acadêmico de universitários do interior do Amazonas-Brasil. Revista Brasileira de Ciências da Saúde, v.20, n.4, p.277-284, 2016.

SZWARCWALD, Celia Landmannet al. Determinantes da autoavaliação de saúde no Brasil e a influência dos comportamentos saudáveis: resultados da Pesquisa Nacional de Saúde, 2013. Revista Brasileira de Epidemiologia, v. 18, p. 33-44, 2015.

WORLD HEALTH ORGANIZATION. Obesity: preventing and managing the global epidemic. World Health Organization, 2000.

Recebido em: 24/04/2019

Aceito em: 13/04/2020 